\title{
Human Fetal Diagnostic Ultrasound Exposimetry System
}

\author{
Dudley Swiney and William D. O'Brien, Jr., \\ Bioacoustics Research Laboratory, Department of Electrical and Computer Engineering, University of Illinois, 405 North Mathews, \\ Urbana, IL 61801
}

\begin{abstract}
Significant improvements have been made to a unique human fetal diagnostic ultrasound exposimetry system [1] which has been designed to measure acoustic pressure in situ with a 7-element linear array hydrophone during an obstetric ultrasound examination. The purpose of the exposimetry system is to develop a data base of fundamental ultrasound propagation properties for human tissue under in vivo conditions from which the ultrasound exposure, and possibly the ultrasound dose, can be estimated under normal clinical conditions. The basic system design allows for the obstetrician to record calibrated acoustic pressure waveforms in situ that have propagated through tissue layers under conditions in which both the diagnostic ultrasound transducer and the calibrated hydrophone are hand-held. The improvements have increased the reliability of the recorded acoustic pressure waveform and provided the new capability of recording the image from the diagnostic ultrasound system simultaneously with the acoustic pressure waveform. The RF acoustic pressure waveforms are digitized at $50 \mathrm{MHz}$ and displayed on a monitor to provide direct visual feedback to the obstetrician. The recorded acoustic pressure waveform is based on a two-decision process. First, the pulse intensity integral (PII), a measure of energy in the pulse, is calculated for each acquired waveform and subsequent recorded waveforms must have a greater PII. Second, a correlation coefficient is calculated for each acquired waveform and must exceed a predetermined correlation coefficient to be recorded. At the same time an $R F$ pressure waveform is acquired, the exposimetry system digitally records the im age from the diagnostic ultrasound scanner. This image shows the location of the hydrophone and provides the basis for estimating the types and distances of tissue layers between the diagnostic ultrasound scanner and the hydrophone, thus providing a direct tie between the acoustic propagation path and the recorded calibrated waveform.
\end{abstract}

\section{INTRODUCTION}

The use of diagnostic ultrasound in obstetrics continues to increase worldwide despite efforts by several national and international organizations [27] and the RADUIS Study [8] to restrict its use to clinically indicated examinations. In fact, in both developed and most second world countries, indiscriminate exposure of the developing embryo and fetus to ultrasound radiation is the norm rather than the exception. Ultrasonic biophysical research has shown that ultrasound can produce changes in living systems [9].

The current scanning conditions and information available to date suggest that the

This work was supported by NIH grant HD 21687 perceived risk associated with the clinical use of ultrasound is low, provided the length of the examination period and methods used for scanning pregnant patients are "prudent" [10]. The FDA [1]12] currently uses a value of $0.3 \mathrm{~dB} / \mathrm{cm}-\mathrm{MHz}$ as a derating factor for manufacturers in their $510(\mathrm{k})$ process (required for all ultrasound systems approved for marketing). It is emphasized, however, that our knowledge regarding ultrasonic bioeffects and biophysical interactions with developing tissues is incomplete at this time. Improved methods for identifying the attenuation that occurs in vivo are required to assess more accurately the "dose" received by the fetus. This information will also help to confirm, or possibly revise, the derating factor (tisssue attenuation coefficient) used by the FDA. The improved exposimetry system is intended to be used in the clinic and operating room to provide a more accurate in situ exposure data base from which "dose" estimates can be made.

\section{PREVIOUS EXPOSIMETRY SYSTEM}

The exposimetry system described previously [1] was developed to measure the acoustic pressure in situ using a calibrated 7-element linear array hydrophone. The 7-element linear array poly(vinylidene difluoride) (PVDF) transducers is positioned near the end of a curved stainless steel tube. Each of the 7 elements are calibrated by a comparison technique against a Marconi membrane hydrophone. The hydrophone is sterilized prior to use and placed as close as possilbe to the tissue of interest using a vaginal approach. The instantaneous pressure waveforms received from the diagnostic ultrasound scanner at the hydrophone are continuously digitized to 8 bits by a $50 \mathrm{MHz}$ A/D converter. These are then transferred to the host $\mathrm{PC}$ via the parallel $\mathrm{I} / \mathrm{O}$ card. The PC's software displays the RF signal from the seven hydrophones with the largest peak compressional pressure on the screen in the same manner that a digital oscilloscope would. This provides direct feedback to the clinician to better position the hydrophone within the tissue. When a waveform is obtained that is larger than any previously recorded, the computer "beeps" and saves the larger waveform to memory. Thus, the largest 
waveform obtained during an exam would be saved to a file for later analysis.

\section{IMPROVED EXPOSIMETRY SYSTEM}

Because of the large success that the original system had in obtaining in situ data, modifications were made to enhance the selection technique for the maximum waveform and to obtain addition information that would link the image received by the ultrasonic transducer to the $\mathrm{RF}$ waveform received by the hydrophone. The specific system improvements were (1) waveform acquisition criteria, (2) display features, and (3) data transfer protocol.

\section{Waveform acquisition criteria}

Previously, the selection criterion for the maximum waveform relied only on the peak value of the instantaneous pressure. To assure that the recorded pressure waveforms are of the highest quality, the system was upgraded with the inclusion of two pressure waveform decision qualifiers. First, the pulse intensity integral (PII) is calculated and compared to the PII value for the previously acquired waveform. PII is calculated using the following equation:

$$
P I I=\int \frac{p^{2}(t)}{\rho c} d t
$$

were $p(t)$ is the instantaneous acoustic pressure, $\rho$ is the density, $c$ is the speed of sound and the integration limits bound the pulse. If the PII value is less than that for the previously acquired waveform,

then the current waveform is rejected; otherwise, the waveform is accepted and replaces the previously acquired waveform, provided that this waveform passes the next level of acceptance test.

For the second level of acceptance, the current waveform is correlated to a wellcharacterized waveform. If the correlation coefficient value is greater than 0.9 , then the current waveform is stored in memory and the second PC is notified, via the serial port, that a maximum waveform has just been recorded.

\section{Display features}

An additional PC was added to the original system to acquire the visual display of the diagnostic ultrasound scanner (Fig. 1). The image is acquired using an 8-bit grayscale frame grabber board. The $60 \mathrm{~Hz}$ video signal from the diagnostic ultrasound scanner is digitized and transferred to the display frame buffer for display with a obtainable update rate of 30 frames per second. Each time this $\mathrm{PC}$ receives a signal from the other PC that a maximum waveform is received at the 7 element hydrophone, the $\mathrm{PC}$ is instructed to save the current frame to memory. The last frame stored in memory is saved as a bitmap for further processing. This bitmap is useful for finding the distance the ultrasound signal traveled and also the thickness of the different layers traversed for a given recorded waveform.

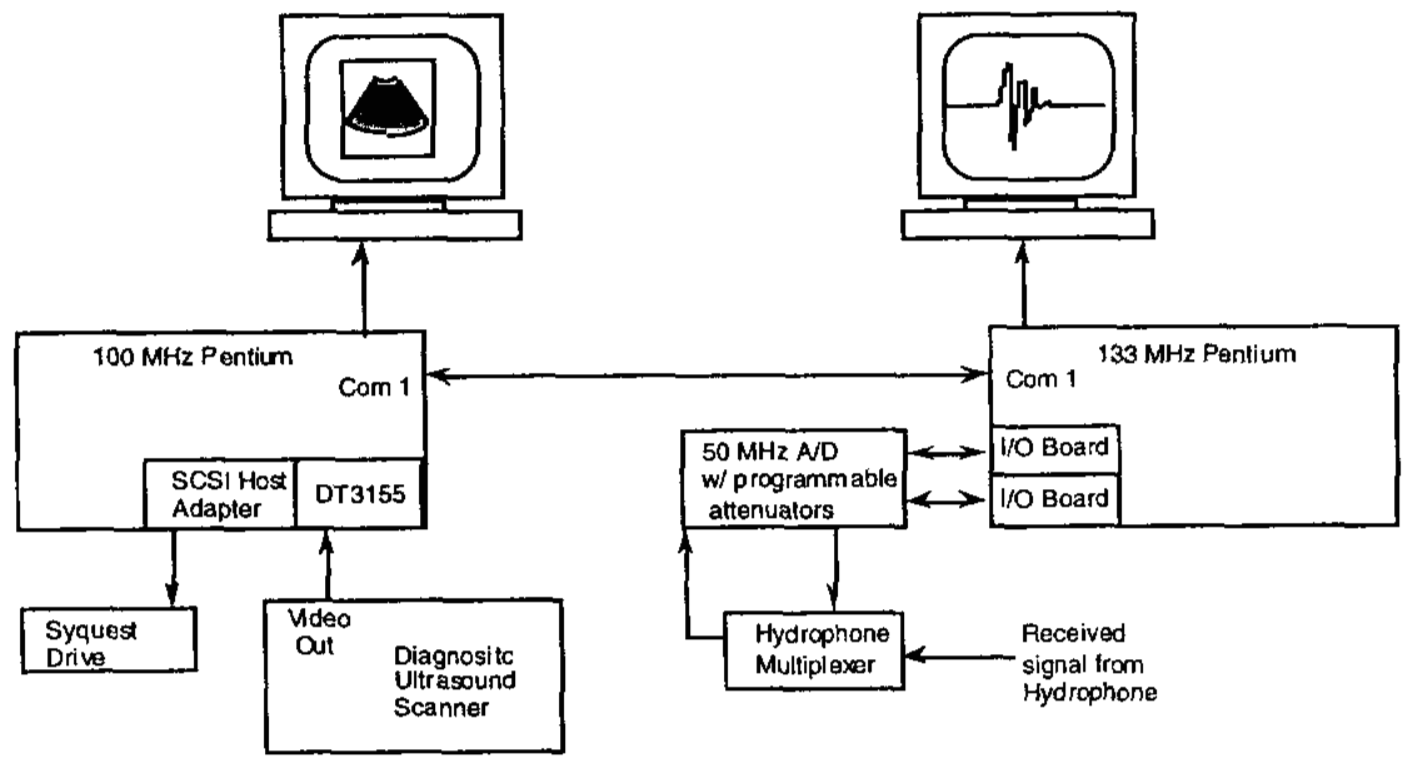

Figure 1. Block diagram of system

Data transfer protocol

All waveforms and images that are recorded are also copied to a $270 \mathrm{MB}$ Syquest ${ }^{\circledR}$ removable drive. This enables the clinician to carry the data back to his office where he also has a Syquest ${ }^{\text {removable drive. }}$ There the clinician indicates on the bimap image the 
boundries of each layer of tissue and the location of the hydrophone using a standard graphics program. He then uses the internet to transfer the data back to the University of Illinois where the analysis is carried out (Fig. 2).
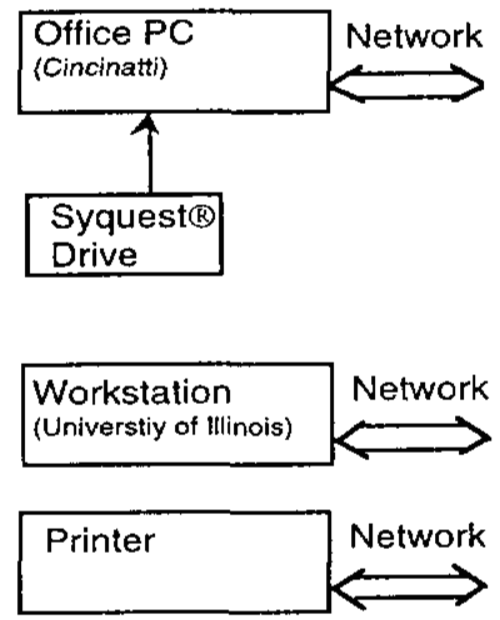

Figure 2 Block diagram of network setup.

\section{TYPICAL PROCESSING PROCEDURES}

Once the data are received from Cincinnati, the data are processed on a Sun SPARCStation/20. A Matlab ${ }^{2}$ script is used to calculate the following values for each patient and combine all the patient data into one file: $I_{\text {SPPA }}$, PII, pulse duration and center frequency. The thickness of each layer is measured on the image that is captured from the output of the diagnostic ultrasound scanner, using the indicators the phyisian placed. From these data it is possible to estimate the propagation speed and insertion loss (attenuation coefficient) of each tissue layer.

\section{CONCLUSION}

The upgraded system will be used to determine separately the in vivo insertion loss of various maternal tissues. These studies will be preformed on patients who are scheduled to undergo a clinically planned, non-emergency cesarean section. After the infant has been delivered and uterine homeostatis has been ensured, the clinician will use this upgraded, custom designed exposimetry system to obtain insertion loss data for (1) the skin and subcutaneous fat, (2) rectus fascia, muscle and peritoneum, (3) and myometrium.

\section{REFERENCES}

1. C. M. W. Daft, T. A. Siddiqi, D. W. Fitting, R. A. Meyer, and W. D. O'Brien, Jr. In Vivo Fetal
Ultrasound Exposimetry. IEEE Transaction on Ultrasonics, Ferroelectrics, and Frequency Control, 37, 500-505, 1990.

2. Environmental Health Criteria 22. World Health Organization, Geneva, Switzerland, 1982.

3. WFUMB Symposium on Safety and Standardization in Medical Ultrasound: Issues and Recommendations Regarding Thermal Mechanisms for Biological Effects of Ultrasound. Barnett, S. B.; Kossoff, G., eds. World Federation for Ultrasound in Medicine and Biology. Ultrasound Med. Biol. 18: 731$814 ; 1992$.

4. Biological Effects of Ultrasound: Mechanisms and Clinical Implications. NCRP Document 74, National Council on Radiation Protection and Measurement, Bethesda, MD, 1984.

5. The Use of Diagnostic Ultrasound Imaging in Pregnancy. National Institute of Child Health and Human Dcvelopment. NIH Consensus Development Conference Process, US Government Printing Office; Washington, DC, 1984.

6. Bioeffects consideration for the safety of diagnostic ultrasound. J. Ultrasound Med. $\underline{7}$ (Suppl):Sl-S38; 1988.

7. Bioeffects and safety of diagnostic ultrasound. American Institute of Ultrasound in Medicine, AIUM Publications, Rockville, MD, 1993.

8. B. G. Ewigman, Crane JP, Frigoletto FD, LeFevre ML, Bain RP, McNellis DG and the RADIUS Study Group. New England J. Med, 329, 821-875, 1993.

9. A. F. Tarantal and W. D. O'Brien, Jr. Discussion of Ultrasonic Safety Related to Obstetrics. In Ultrasound Applied to Obstetrics and Gynecology, 3rd Edition, Rudy E. Sabbagha, ed., J. B. Lippincott Co., Philadelphia, PA, pp 4556, 1994.

10. M. C. Ziskin and D. B. Petitti. Epidemiology of human exposure to ultrasound: A critical review. Ultrasound Med Biol, 14, 91-96, 1988.

11." $501(\mathrm{k})$ Guide for Measuring and Reporting Acoustic Output of Diagnostic Ultrasound Medical Devices, December, 1985," Center for Devices and Radiological Health, US Food and Drug Administration, Rockville, MD, 1985.

12. "Revised 510(k) Diagnostic Ultrasound Guidance for 1993." Center for Devices and Radiological Health, US Food and Drug Administration, Rockville, MD February 17, 1993. 